\title{
ON UNIFORM ELEMENTARY ESTIMATES OF ARITHMETIC SUMS
}

\author{
STEFAN A. BURR
}

\begin{abstract}
A simple, elementary method is given for deriving estimates of sums of arithmetic functions, the estimates being in a certain sense uniform over a class of functions appearing in the summation. The method is particularly well suited to estimates needed in applications of Selberg's sieve.
\end{abstract}

Bateman [1] and Tull ([2], [3]) have given an elementary, but very useful, method of estimating sums of arithmetic functions by means of convolution arguments. It is surprising that such a straightforward and useful technique was not systematically developed earlier. In fact, the idea goes at least as far back as 1921, when Wilson [4] used it to estimate $\sum_{n \leqq x} d^{2}(n)$, conjecturing that Ramanujan had done the same. However, Wilson did not apparently recognize that the idea had very wide applicability. Bateman and Tull seem to have been the first to recognize the very general usefulness of the technique, and Tull ([2], [3]) has developed the method very fully.

In this paper we will use a variant of the method to derive estimates of sums of arithmetic functions, the estimates being in a certain sense uniform over a class of functions appearing in the summation. This sort of problem is particularly prevalent in applications of Selberg's sieve, where the functions appearing tend to be rather complicated. Fortunately, the complexities involved are of little consequence in applying the method.

In applying Selberg's sieve, the estimates required are of a rather special type, and it is often sufficient to have estimates that are fairly crude. In fact, it can be highly desirable to have straightforward estimates that can be easily applied. With this in mind, we will restrict ourselves to rather simple results; however, it should be obvious that they can be greatly generalized. By restricting ourselves to simple results, we may help to accomplish another objective of this paper, namely to render the important idea involved more accessible. (Tull's development is so

Presented to the Society, January 24, 1969 under the title An elementary method for estimating number-theoretic sums; received by the editors June 6, 1972.

AMS (MOS) subject classifications (1970). Primary 10H25; Secondary 10A20, $10 \mathrm{H} 30$.

(C) American Mathematical Society 1973 
thorough as to obscure the essential simplicity of the idea; each of [2] and [3] have one main theorem, whose statements occupy one and two pages respectively.)

Before proceeding to the results, we adopt the following convention. If the letters $f$ and $g$ denote arithmetic functions, then $F(s), G(s), G_{1}(s)$, and $G_{2}(s)$ denote the Dirichlet series

$$
\begin{gathered}
\sum_{n=1}^{\infty} \frac{f(n)}{n^{s}}, \sum_{n=1}^{\infty} \frac{g(n)}{n^{s}}, \\
\sum_{n=1}^{\infty} \frac{|g(n)|}{n^{s}}, \\
|g(1)|+\sum_{n=2}^{\infty} \frac{|g(n)| \log n}{n^{s}},
\end{gathered}
$$

respectively. We will also define $\tau_{r}(n)$ to be the number of ways $n$ can be expressed as a product of $r$ factors (taking order into account). We use $\tau$ instead of the more usual $d$ so that $d$ can be used as an index. Note that $\zeta^{r}(s)$ is the Dirichlet series generating function of $\tau_{r}$. We will use the following crude forms of well-known results:

$$
\begin{aligned}
& \sum_{n \leqq x} \tau_{r}(n)=\frac{1}{(r-1) !} x \log ^{r-1} x+O\left(x \log ^{r-2} x\right), \\
& \sum_{n \leqq x} \frac{\tau_{r}(n)}{n}=\frac{1}{r !} \log ^{r} x+O\left(\log ^{r-1} x\right) .
\end{aligned}
$$

For an elementary proof of a much stronger version of (1) see [5, p. 263]; (2) follows from (1) by Abel summation.

THEOREM 1. Let $f$ be an arithemetic function and let $g$ be such that

$$
f(n)=\sum_{d \mid n} g(d) \tau_{r}(n / d)
$$

with $r \geqq 2$, and suppose that $G_{2}(1)$ exists. Then

$$
\sum_{n \leqq x} f(n)=\frac{1}{(r-1) !} G(1) x \log ^{r-1} x+O\left(G_{2}(1) x \log ^{r-2} x\right)
$$

and

$$
\sum_{n \leqq x} \frac{f(n)}{n}=\frac{1}{r !} G(1) \log ^{r} x+O\left(G_{2}(1) \log ^{r-1} x\right),
$$

where the implied constants depend only on $r$. 
Proof. We prove (4) first. Making use of (3) and (1), we have

$$
\begin{aligned}
\sum_{n \leqq x} f(n)= & \sum_{d m \leqq x} g(d) \tau_{r}(m)=\sum_{d \leqq x} g(d) \sum_{m \leqq x / d} \tau_{r}(m) \\
= & \sum_{d \leqq x} g(d)\left(\frac{1}{(r-1) !} \frac{x}{d} \log ^{r-1} \frac{x}{d}+O\left(\frac{x}{d} \log ^{r-2} x\right)\right) \\
= & x \sum_{d \leqq x} \frac{g(d)}{d}\left(\frac { 1 } { ( r - 1 ) ! } \left(\log ^{r-1} x-(r-1) \log ^{r-2} x \log d\right.\right. \\
& \left.\left.+\cdots+(-1)^{r-1} \log ^{r-1} d\right)+O\left(\log ^{r-2} x\right)\right) \\
= & \frac{1}{(r-1) !} x \log ^{r-1} x \sum_{d \leq x} \frac{g(d)}{d}+O\left(x \log ^{r-2} x \sum_{d \leqq x} \frac{|g(d)|}{d}(\log d+1)\right) \\
= & \frac{1}{(r-1) !} x \log ^{r-1} x \sum_{d=1}^{\infty} \frac{g(d)}{d}+O\left(x \log ^{r-2} x \sum_{d>x} \frac{|g(d)| \log d}{d}\right) \\
& +O\left(x \log ^{r-2} x\left(|g(1)|+\sum_{d \leqq x} \frac{|g(d)| \log d}{d}\right)\right) \\
= & \frac{1}{(r-1) !} G(1) x \log ^{r-1} x+O\left(G_{2}(1) x \log ^{r-2} x\right),
\end{aligned}
$$

where it is clear the implied constant depends only on $r$.

The result (5) can be proved from (4) using Abel summation; but it can also be proved in much the same manner as (4), using (3) and (2):

$$
\begin{aligned}
\sum_{n \leqq x} \frac{f(n)}{n}= & \sum_{d \leqq x} \frac{g(d)}{d} \sum_{m \leqq x / d} \frac{\tau_{r}(m)}{m} \\
= & \sum_{d \leqq x} \frac{g(d)}{d}\left(\frac{1}{r !} \log ^{r} \frac{x}{d}+O\left(\log ^{r-1} x\right)\right) \\
= & \frac{1}{r !} \log ^{r} x \sum_{d \leqq x} \frac{g(d)}{d}+O\left(\log ^{r-1} x \sum_{d \leqq x} \frac{|g(d)|}{d}(\log d+1)\right) \\
= & \frac{1}{r !} G(1) \log ^{r} x+O\left(\log ^{r-1} x \sum_{d>x} \frac{|g(d)| \log d}{d}\right) \\
& +O\left(\log ^{r-1} x\left(|g(1)|+\sum_{d \leqq x}^{|g(d)| \log d} \frac{1}{d}\right)\right) \\
= & \frac{1}{r !} G(1) \log ^{r} x+O\left(G_{2}(1) \log ^{r-1} x\right) .
\end{aligned}
$$


Again, the implied constant clearly depends only on $r$, so the proof is complete.

In order to apply the above theorem to a function $f$, it is necessary to determine a relation (3). This can be done by means of an inversion formula, but at least in the case of a multiplicative function, it seems most convenient to work directly with Dirichlet series. If $F(s)=G(s) \zeta^{r}(s)$, then it is clear that the $g$ so determined will satisfy (3). If $G_{2}(1)$ exists, then the theorem can be applied, and in fact $g$ need not be explicitly determined, since the result requires only $G(1)$ and $G_{2}(1)$. These facts are illustrated by the following theorem, which gives an estimate of the sort typical of those needed in applications of Selberg's sieve.

THEOREM 2. If $r \geqq 2$ and $N \geqq 1$ are integers,

$$
\sum_{n \leqq x ;(n, N)=1} \frac{\mu^{2}(n) \tau_{r}(n)}{n}=C(N) \log ^{r} x+O\left(\log ^{r-1} x \log ^{r+1} \log 3 N\right),
$$

where the implied constant depends only on $r$, and where

$$
C(N)=\prod_{p \mid N}(1+r / p)^{-1} \cdot \prod_{p}(1-1 / p)^{r}(1+r / p) .
$$

Here the second product runs over all primes.

Proof. Let $\chi_{N}(n)$ be 1 if $(n, N)=1$, and 0 otherwise. Then if we take $f(n)=\mu^{2}(n) \chi_{N}(n) \tau_{r}(n)$, the sum can be written $\sum_{n \leqq x} f(n) / n$. We then have, expressing $F(s)$ as an Euler product:

$$
\begin{aligned}
F(s) & =\prod_{p}\left(1+\chi_{N}(p) r p^{-s}\right)=\prod_{p \nmid N}\left(1+r p^{-s}\right) \\
& =\zeta^{r}(s) \prod_{p}\left(1-p^{-s}\right)^{r} \prod_{p \nmid N}\left(1+r p^{-s}\right) \\
& =\zeta^{r}(s) \prod_{p}\left(1-p^{-s}\right)^{r}\left(1+r p^{-s}\right) \prod_{p^{\prime} N^{*}}\left(1+r p^{-s}\right)^{-1}=\zeta^{r}(s) G(s) .
\end{aligned}
$$

This defines $G$ and hence the function $g$. Note that the form of the Euler product for $F$ made it clear what power of the zeta function should be removed from $F$. We now must estimate $G_{2}(1)$. To this end we rewrite $G$ :

so that

$$
\begin{aligned}
G(s) & =\prod_{p \mid N}\left(1-p^{-s}\right)^{r} \prod_{p \nmid N}\left(1-p^{-s}\right)^{r}\left(1+r p^{-s}\right) \\
& =\prod_{p \mid N}\left(1-p^{-s}\right)^{r} \prod_{p \nmid N}\left(1-\frac{1}{2} r(r+1) p^{-2 s}+\cdots\right),
\end{aligned}
$$

$$
G_{1}(s)=\prod_{p \mid N}\left(1+p^{-s}\right)^{r} \prod_{p \nmid N}\left(1+\frac{1}{2} r(r+1) p^{-2 s}+\cdots\right)=H(s) K(s) .
$$


Now

$$
G_{2}(1)=1-G_{1}^{\prime}(1)=1-\left(H^{\prime}(1) K(1)+H(1) K^{\prime}(1)\right)=O\left(-H^{\prime}(1)+H(1)\right),
$$

since $K(1)$ and $K^{\prime}(1)$ are bounded independently of $N$.

We have $-H^{\prime}(s)=r H(s) \sum_{p \mid N}\left(p^{-s} \log p /\left(1+p^{-s}\right)\right)$, so

$$
-H^{\prime}(1)+H(1)=O\left(\prod_{p \mid N}(1+1 / p)^{r}\left(1+\sum_{p \mid N} \frac{\log p}{p}\right)\right) .
$$

From standard estimates involving primes, for instance see [6, Chapter XXII], one can deduce $\prod_{p \mid N}(1+1 / p)=O(\log \log 3 N)$ and

Therefore

$$
1+\sum_{p \mid N} \frac{\log p}{p}=O(\log \log 3 N) \text {. }
$$

$$
G_{2}(1)=O\left(\log ^{r+1} \log 3 N\right) .
$$

Using the fact that (6) implies a relation of the sort (3), and observing that $G(1)=C(N)$, the second formula in Theorem 1 yields

$$
\sum_{n \leqq x} f(n) / n=C(N) \log ^{r} x+O\left(\log ^{r-1} x \log ^{r+1} \log 3 N\right),
$$

which is the desired result.

It is easy to see that $1 / C(N)=O\left(\log ^{r} \log 3 N\right)$, so that the second term is truly of lower order than the first whenever, for instance, $N$ is no greater than a power of $x$. Theorem 2 permits one to derive, using Selberg's sieve, a fairly simple universal upper bound for the number of $n \leqq M$ for which $n+t_{1}, \cdots, n+t_{r}$ are all primes. This bound in turn provides the starting point for an elementary solution of the Waring-Goldbach problem. For details see [7].

Theorem 1, and the technique used in Theorem 2, can replace a number of intricate arguments that have appeared in the literature, for instance those of [8] and [9, Chapter 6]. Although some effort is still required, it is quite mechanical in nature.

It should be noted that Theorem 1 can be easily extended to results with more principal terms and an error term of similar form. Moreover, with a little work, a bound on the error term can be given explicitly. For example, if $r=2$, the right-hand side of (4) can be readily replaced by

$$
G(1) x \log x+\left(G^{\prime}(1)+(2 \gamma-1) G(1)\right) x+E(x),
$$

with $|E(x)| \leqq 8 G_{2}(\alpha) x^{\alpha}$ for all $x \geqq 1$ and all $\alpha \leqq 1$ for which $G_{2}(\alpha)$ exists. 


\section{REFERENCES}

1. P. T. Bateman, Proof of a conjecture of Grosswald, Duke Math. J. 25 (1958), 67-72. MR 19, 1040.

2. J. P. Tull, Dirichlet multiplication in lattice point problems, Duke Math. J. 26 (1959), 73-80. MR 21 \#659.

3. - Dirichlet multiplication in lattice point problems. IJ, Pacific J. Math. 9 (1959), 609-615. MR 21 \#6362.

4. B. M. Wilson, Proofs of some formulae enunciated by Ramanujan, Proc. London Math. Soc. (2) 21 (1922), 235-255.

5. E. C. Titschmarsh, The theory of the Riemann zeta-function, 2nd ed., Clarendon Press, Oxford, 1951. MR 13, 741.

6. G. H. Hardy and E. M. Wright, An introduction to the theory of numbers, 4th ed., Oxford Univ. Press, London, 1960.

7. S. A. Burr, An elementary solution of the Waring-Goldbach problem (to appear).

8. H. N. Shapiro and J. Warga, On the representation of large integers as sums of primes. I, Comm. Pure Appl. Math. 3 (1950), 153-176. MR 12, 244.

9. A. O. Gel'fond and Ju. V. Linnik, Elementary methods in the analytic theory of numbers, Fizmatgiz, Moscow, 1962; English transl., Rand McNally, Chicago, Ill., 1965; Pergamon Press, New York, 1966. MR 32 \#5575a, b; 34 \#1252.

Bell Telephone Laboratories, Incorporated, Madison, New Jersey 07940 